\title{
Inference of Restricted Stochastic Boolean GRN's by Bayesian Error and Entropy Based Criteria
}

\author{
David Correa Martins Jr. ${ }^{1}$, Evaldo Araújo de Oliveira ${ }^{2}$, \\ Vitor Hugo Louzada ${ }^{2}$, and Ronaldo Fumio Hashimoto ${ }^{2}$ \\ ${ }^{1}$ Center for Mathematics, Computation and Cognition - Federal University of ABC, \\ Brazil \\ david.martins@ufabc.edu.br \\ 2 Institute of Mathematics and Statistics - University of São Paulo, Brazil \\ \{evaldo, louzada, ronaldo\}@vision.ime.usp.br
}

\begin{abstract}
This work compares two frequently used criterion functions in inference of gene regulatory networks (GRN), one based on Bayesian error and another based on conditional entropy. The network model utilized was the stochastic restricted Boolean network model; the tests were realized in the well studied yeast cell-cycle and in randomly generated networks. The experimental results support the use of entropy in relation to the use of Bayesian error and indicate that the application of a fast greedy feature selection algorithm combined with an entropy-based criterion function can be used to infer accurate GRN's, allowing to accurately infer networks with thousands of genes in a feasible computational time cost, even though some genes are influenced by many other genes.
\end{abstract}

Keywords: feature selection, gene regulatory networks inference, stochastic restricted Boolean network models, entropy, Bayesian error.

\section{Introduction}

Gene regulatory networks (GRN) models help us to study biological phenomena (e.g. cell cycle) and diseases (e.g. cancer). Therefore, the inference of such networks is an important problem to be addressed. Unfortunately, the GRN inference problem usually involves data with a large number of variables and small number of observations, making the problem particularly hard due to error estimation issues. In this context, many inference algorithms have been proposed. A survey of GRN inference methods can be found in [1].

In this context, we compare two feature selection criteria commonly used for inference of gene regulatory networks, one based on Bayesian error (non-linear coefficient of determination) and another based on mean conditional entropy (uncertainty coefficient). The experiments considered the stochastic model of the yeast cell cycle provided by Zhang et al, which is based on restricted Boolean networks [2]. Besides the network topology of the yeast cell cycle model, other randomly generated topologies following a similar parametrization were included

I. Bloch and R.M. Cesar, Jr. (Eds.): CIARP 2010, LNCS 6419, pp. $144-152,2010$.

(C) Springer-Verlag Berlin Heidelberg 2010 
in the experiments. Applying exhaustive search and a classical and fast feature selection algorithm (Sequential Forward Selection - SFS) to the transition matrices corresponding to such networks, it can be observed a significantly better performance of the entropy over the Bayesian error in recovering the groundtruth connections. Besides, the application of SFS guided by the entropy based criterion leaded to surprisingly good results, which is a valid alternative to become feasible the inference of GRNs considering thousands of genes with nice quality. These findings are the main contribution of this work.

Next section presents a brief background on feature selection, the algorithms and criterion functions used. In Section 3, the restricted Boolean network model of the yeast cell-cycle is presented. Section 4 discusses the GRN inference results obtained by the application of the considered algorithms criterion functions. Finally, the conclusion of this work along with future perspectives are found in Section 5

\section{Feature Selection}

Feature selection techniques are composed by two main parts: a search algorithm and a criterion function that guides the algorithm. In feature selection, the only way to guarantee that the optimal solution be achieved is by exploring all possible subsets of all dimensions (exhaustive search), although depending on the behavior of the criterion function, it is possible to design branch-and-bound algorithms that obtain the optimal solution without the need to investigate the whole space of solutions [3,4]. Greedy algorithms like Best Individual Features or Sequential Forward Selection (SFS) are very fast, although do not guarantee the optimal solution due to the nesting effect, i.e. a phenomenon in which the inclusion of the best features according to a given criterion may not lead to the optimal subset [5]. Yet, this phenomenon can be explained by the intrinsically multivariate prediction concept [6], also known as synergy [7], which states that it is possible to obtain a very good predictor set with regard to a considered target even that all its properly contained subsets do not offer any prediction about the target. Due to the nesting effect, many floating search algorithms that try to alleviate it by trying to successively add elements to and remove elements from the current solution have been proposed.

Here the attention is given to the SFS, a genuinely greedy feature selection algorithm [8]. It starts with the empty set and includes the best feature according to the criterion function. In every step $i$, it adds the $i$-th feature that forms the best set with the already included $i-1$ features in the partial solution. This process continues until reaching a stop condition, which usually either is based on a fixed dimensionality given as input or based on the evaluation of the improvement of the criterion function (if the improvement by adding the next feature is smaller than a certain threshold, it stops).

With regard to the criterion functions, the focus is given on two commonly used criteria in GRN inference. One of them is the coefficient of non-linear determination $(\mathrm{CoD})$, which considers the error committed by a subset in classifying 
the target value (Bayesian error) $[9,10,11$. The $\mathrm{CoD}$ of the target $\mathrm{Y}$ given the knowledge of $\mathbf{X}=\left(X_{1}, \ldots, X_{n}\right)$ is given by:

$$
C o D_{Y}(\mathbf{X})=\frac{\varepsilon(Y)-\varepsilon(Y \mid \mathbf{X})}{\varepsilon(Y)}
$$

where $\varepsilon(Y)=1-\max _{y \in Y} P(y)$ is the prior error, i.e. the error by predicting $Y$ in the absence of other observations, and $\varepsilon(Y \mid \mathbf{X})=\sum_{\mathbf{x} \in \mathbf{X}} P(\mathbf{x})(1-$ $\left.\max _{y \in Y} P(y \mid \mathbf{x})\right)$ is the average error by predicting $Y$ based on the observation of $\mathbf{X}$.

Criterion functions based on entropy $(H)$, such as mutual information or mean conditional entropy, are frequently adopted for GRN inference as well [12, 13. The Uncertainty Coefficient (UC) is similar to CoD, but instead of using Bayesian error, it employs entropy [14. Its equation is given by:

$$
U C_{Y}(\mathbf{X})=\frac{H(Y)-H(Y \mid \mathbf{X})}{H(Y)}
$$

where $H(Y)=-\sum_{y \in Y} P(y) \log P(y)$ is the entropy of predicting $Y$ in the absence of other observations, and $H(Y \mid \mathbf{X})=\sum_{\mathbf{x} \in \mathbf{X}} P(\mathbf{x}) H(Y \mid \mathbf{x})$ is the mean conditional entropy of $Y$ given the observation of $\mathbf{X}$.

\section{Yeast Cell-Cycle Stochastic Restricted Boolean Model}

Following the yeast cell-cycle model with 11 genes proposed by Li et al [15] and its stochastic version [2, a Restricted Boolean Network is defined as a graph where each node is represented by a Boolean variable $s_{i} \in\{0,1\}$ and its dynamics is given by the following transition probabilities:

$$
\begin{array}{r}
P\left(s_{1}(t+1), s_{2}(t+1) \ldots s_{11}(t+1) \mid s_{1}(t), s_{2}(t) \ldots s_{11}(t)\right)= \\
\prod_{i} P\left(s_{i}(t+1) \mid s_{1}(t), s_{2}(t) \ldots s_{11}(t)\right),
\end{array}
$$

where

$$
\begin{aligned}
& P\left(s_{i}(t+1)=\sigma_{i} \mid s_{1}(t), \ldots s_{11}(t)\right)=\frac{\exp \left\{-\beta\left(1-2 \sigma_{i}\right) \sum_{j} w_{i j} s_{j}\right\}}{\exp \left\{-\beta \sum_{j} w_{i j} s_{j}\right\}+\exp \left\{\beta \sum_{j} w_{i j} s_{j}\right\}}, \\
& \text { if } \sum_{j} w_{i j} s_{j} \neq 0 \text { or } \\
& \qquad P\left(s_{i}(t+1)=s_{i}(t) \mid s_{1}(t), \ldots s_{11}(t)\right)=1 /(1+\exp \{-\alpha\})
\end{aligned}
$$

otherwise.

In the context of gene regulatory networks, $s_{i}(t)$ represents the expression of gene $i$ at the moment $t ; w_{i j}$ sets the influence of gene $i$ on gene $j\left(w_{i j} \in\{-1,0,1\}\right.$ for $i \neq j$ and $w_{i j} \in\{-0.1,0\}$ for $i=j$ ). The parameters $\alpha$ and $\beta$ are positive and related to intrinsic and input noises, respectively. They work as inverse temperatures, i.e. the probability of the system to follow the deterministic pathway increases with their values. These deterministic pathways are the trajectories of the system in the state space of the noiseless case. In the Zhang et al model, 
these pathways are the trajectories of the network state in the deterministic yeast cell-cycle model [15].

For a network with $N$ nodes, there are $2^{N}$ distinct states. Naturally, some of these states are not allowed in the dynamics of the deterministic model (noiseless case). But in the presence of noise, the system eventually can be found in some of the "forbidden" states.

The model proposed by Equations 4 and 5 comes from the assumption in which all nodes are under the same kind of noise and that the transition function does not change in time, which means that it is a time homogeneous Markovian process. Besides, as can be noted, all states are accessible, i.e. the Markov chain is ergodic and, therefore, there is a time-invariant probability distribution $\pi=$ $\left(\pi_{0}, \pi_{1}, \ldots, \pi_{2^{N}-1}\right)$ to which the system converges:

$$
\lim _{r \rightarrow \infty} p_{m n}(r)=\pi_{n}, \quad 0 \leqslant m, n<2^{N}
$$

where $p_{m n}(r)$ is the element on the row $m$ and column $n$ of $P^{r}$, with $P$ being the transition matrix defined by Eq. 3 .

\section{Experimental Results}

Using the model described in Section 3, we have all state transition probabilities of the Markov chain process given in a matrix $P$. As this is an ergodic matrix (see Section 3), we have the limit probability distribution of all states given by the vector $\pi$. With $P$ and $\pi$, it is possible to obtain the joint probability distribution table (JPD) for all possible subsets of variables, allowing to apply criterion functions to evaluate any subset of genes as predictor candidates for any gene considered as target [16].

Here we considered two feature selection algorithms: the exhaustive search (which examines all possible subsets of all dimensions, returning the optimal subset with the smallest dimension) and the SFS (see Section 2 for a brief description) with a stop condition that is based on the improvement of the criterion (if the addition of the $n$-th gene does not improve the result obtained by the current subset of dimension $n-1$, the process returns the last one as result). Each algorithm was applied considering two criterion functions: CoD (Bayesian error based, see Equation 11) and UC (entropy based, see Equation 2). Therefore, four methods were considered for comparison: exhaustive search with CoD (ES-CoD), exhaustive search with UC (ES-UC), SFS with CoD (SFS-CoD) and SFS with UC (SFS-UC).

In the first experiment, we set the $w_{i j}$ 's as the Zhang et al model [2] and applied the four methods aforementioned to the inference of the network in the case of small $(\alpha=5, \beta=6)$ and large temperature $(\alpha=0.05, \beta=0.06)$. The accuracy was perfect in both cases, i.e. the network was fully recovered, presenting neither false positives nor false negatives for all methods used (ESCoD, ES-UC, SFS-CoD and SFS-UC).

In order to compare the inference performance obtained by the $\mathrm{CoD}$ and the $\mathrm{UC}$ criteria in more situations, we generate random networks with a parametrization close to that presented by the cell-cycle network of the budding yeast using 
Table 1. Tables containing the numbers of false negatives (FN) and false positives (FP) obtained by the application of ES-CoD, ES-UC, SFS-CoD and SFS-UC to infer each one of the 10 randomly generated topology samples considering small temperature $(\alpha=5, \beta=6)$ and large temperature $(\alpha=0.05, \beta=0.06)$.

\begin{tabular}{|c|c|}
\hline$\alpha$ & $\beta$ \\
\hline 5 & 6 \\
\hline 0.05 & 0.06 \\
\hline
\end{tabular}

\begin{tabular}{|c|c|c|c|c|c|c|c|}
\hline \multicolumn{7}{|c|}{ Sample 1 } \\
\hline ES-CoD & ES-UC & SFS-CoD & \multicolumn{2}{|c|}{ SFS-UC } \\
\hline FN & FP & FN & FP & FN & FP & FN & FP \\
\hline 12 & 0 & 3 & 0 & 12 & 0 & 3 & 0 \\
\hline 0 & 0 & 0 & 0 & 12 & 0 & 0 & 0 \\
\hline
\end{tabular}

\begin{tabular}{|c|c|c|c|c|c|c|c|}
\hline \multicolumn{7}{|c|}{ Sample 3 } \\
\hline ES-CoD & ES-UC & \multicolumn{2}{|c|}{ SFS-CoD } & \multicolumn{2}{|c|}{ SFS-UC } \\
\hline FN & FP & FN & FP & FN & FP & FN & FP \\
\hline 3 & 0 & 0 & 0 & 8 & 0 & 0 & 0 \\
\hline 3 & 0 & 0 & 0 & 17 & 0 & 0 & 0 \\
\hline
\end{tabular}

\begin{tabular}{|c|c|c|c|c|c|c|c|}
\hline \multicolumn{7}{|c|}{ Sample 5 } \\
\hline ES-CoD & ES-UC & \multicolumn{2}{|c|}{ SFS-CoD } & \multicolumn{2}{|c|}{ SFS-UC } \\
\hline FN & FP & FN & FP & FN & FP & FN & FP \\
\hline 0 & 0 & 0 & 0 & 8 & 0 & 0 & 1 \\
\hline 0 & 0 & 0 & 0 & 18 & 0 & 0 & 0 \\
\hline
\end{tabular}

\begin{tabular}{|c|c|c|c|c|c|c|c|}
\hline \multicolumn{7}{|c|}{ Sample 7} \\
\hline ES-CoD & ES-UC & \multicolumn{1}{l|}{ SFS-CoD } & \multicolumn{2}{l|}{ SFS-UC } \\
\hline FN & FP & FN & FP & FN & FP & FN & FP \\
\hline 14 & 0 & 11 & 0 & 17 & 0 & 11 & 0 \\
\hline 0 & 0 & 0 & 0 & 22 & 0 & 0 & 0 \\
\hline
\end{tabular}

\begin{tabular}{|c|c|c|c|c|c|c|c|}
\hline \multicolumn{10}{|c|}{ Sample 9 } \\
\hline ES-CoD & ES-UC & \multicolumn{3}{|l|}{ SFS-CoD } & \multicolumn{2}{|c|}{ SFS-UC } \\
\hline FN & FP & FN & FP & FN & FP & FN & FP \\
\hline 30 & 0 & 18 & 0 & 30 & 0 & 18 & 0 \\
\hline 5 & 0 & 0 & 0 & 20 & 0 & 0 & 0 \\
\hline
\end{tabular}

\begin{tabular}{|c|c|c|c|c|c|c|c|}
\hline \multicolumn{7}{|c|}{ Sample 2 } \\
\hline ES-CoD & ES-UC & \multicolumn{2}{|c|}{ SFS-CoD } & \multicolumn{2}{|c|}{ SFS-UC } \\
\hline FN & FP & FN & FP & FN & FP & FN & FP \\
\hline 0 & 0 & 0 & 0 & 7 & 0 & 0 & 1 \\
\hline 0 & 0 & 0 & 0 & 17 & 0 & 0 & 0 \\
\hline
\end{tabular}

\begin{tabular}{|c|c|c|c|c|c|c|c|}
\hline \multicolumn{7}{|c|}{ Sample 4} \\
\hline ES-CoD & ES-UC & \multicolumn{2}{|c|}{ SFS-CoD } & \multicolumn{2}{|c|}{ SFS-UC } \\
\hline FN & FP & FN & FP & FN & FP & FN & FP \\
\hline 0 & 0 & 0 & 0 & 3 & 0 & 0 & 0 \\
\hline 0 & 0 & 0 & 0 & 20 & 0 & 0 & 0 \\
\hline
\end{tabular}

\begin{tabular}{|c|c|c|c|c|c|c|c|}
\hline \multicolumn{7}{|c|}{ Sample 6} \\
\hline ES-CoD & ES-UC & \multicolumn{2}{|c|}{ SFS-CoD } & \multicolumn{3}{|c|}{ SFS-UC } \\
\hline FN & FP & FN & FP & FN & FP & FN & FP \\
\hline 1 & 0 & 0 & 0 & 12 & 0 & 0 & 0 \\
\hline 0 & 0 & 0 & 0 & 18 & 0 & 0 & 0 \\
\hline
\end{tabular}

\begin{tabular}{|c|c|c|c|c|c|c|c|}
\hline \multicolumn{7}{|c|}{ Sample 8} \\
\hline ES-CoD & ES-UC & SFS-CoD & \multicolumn{2}{l|}{ SFS-UC } \\
\hline FN & FP & FN & FP & FN & FP & FN & FP \\
\hline 0 & 0 & 0 & 0 & 2 & 0 & 0 & 0 \\
\hline 4 & 0 & 0 & 0 & 9 & 0 & 0 & 0 \\
\hline
\end{tabular}

\begin{tabular}{|c|c|c|c|c|c|c|c|}
\hline \multicolumn{10}{|c|}{ Sample 10 } \\
\hline \multicolumn{2}{|c|}{ ES-CoD } & ES-UC & \multicolumn{2}{l|}{ SFS-CoD } & \multicolumn{3}{l|}{ SFS-UC } \\
\hline FN & FP & FN & FP & FN & FP & FN & FP \\
\hline 18 & 0 & 13 & 0 & 18 & 0 & 13 & 0 \\
\hline 0 & 0 & 0 & 0 & 25 & 0 & 0 & 0 \\
\hline
\end{tabular}

the model given by Equation 3. For each generated network, the connection matrix $w$ is randomly set in the following way. Firstly, as occurs with the yeast model, we consider $N=11$ genes, fixing one of the genes (gene 1 ) as the source of the network (input degree equals to zero) by making $w_{11}=w_{21}=\ldots=w_{N 1}=0$. As there are 29 connections present in yeast model, 30 cells from $w_{i j}, 1 \leq i, j \leq N$ are randomly chosen. For these randomly chosen cells, each one has a probability of 0.5 to be represented by activation $(+1)$ or inhibition $(-1)$, while all other cells remain zero. Also, considering that in yeast model, 4 out of 11 genes suffer self-degradation (approximately one third), each gene has probability $\frac{1}{3}$ to be self-degradated. If a gene $i$ suffers self-degradation, then $w_{i i}=-0.1$.

We applied the random network generation procedure presented above to generate 10 random network topology samples. For each network topology, the 
transition matrix $P$ and the limit distribution $\pi$ were computed considering both large temperature $(\alpha=5$ and $\beta=6)$ and small temperature $(\alpha=0.05$ and $\beta=0.06)$. Finally, these transition matrices and limit distributions were supplied as inputs to the four inference methods considered (ES-CoD, ES-UC, SFS-CoD, SFS-UC). The numbers of false negatives (FN) and false positives (FP) obtained for each method applied to each considered sample are shown in Table 1. Table 2 summarizes these results presenting the mean and standard deviation values.

Table 2. Averages and standard deviations of the results presented in Table 1

\begin{tabular}{|c|c|c|c|c|c|c|c|c|}
\hline & \multicolumn{3}{|c|}{ ES-CoD } & \multicolumn{3}{|c|}{ ES-UC } & \multicolumn{3}{|c|}{ SFS-CoD } & \multicolumn{2}{|c|}{ SFS-UC } \\
\cline { 2 - 9 } & FN & FP & FN & FP & FN & FP & FN & FP \\
\hline$(\alpha=5, \beta=6)$ averages & 7.8 & 0 & 4.5 & 0 & 11.7 & 0 & 4.5 & 0.2 \\
$(\alpha=5, \beta=6)$ std. dev. & 10.4 & 0 & 6.8 & 0 & 8.3 & 0 & 6.8 & 0.4 \\
\hline$(\alpha=0.05, \beta=0.06)$ averages & 1.2 & 0 & 0 & 0 & 17.8 & 0 & 0 & 0 \\
$(\alpha=0.05, \beta=0.06)$ std. dev. & 2.0 & 0 & 0 & 0 & 4.6 & 0 & 0 & 0 \\
\hline
\end{tabular}

The first important observation that can be drawn from Tables 1 and 2 is that the inference accuracy of the uncertainty coefficient for both exhaustive search and SFS is significantly better than the accuracy obtained by the application of CoD. In fact, CoD performs very poorly especially when embedded in the SFS algorithm, since in average, the percentage of recovery is around $50 \%$ (15 out of 30 ) of the connections. Moreover, for the sample 9 considering $\alpha=5$ and $\beta=6$, CoD was incapable to identify any connection even when exhaustive search was applied (i.e., CoD was zero for all possible subsets of all genes considered as targets). On the other hand, SFS performed nicely when guided by the uncertainty coefficient, having a very small number of false negatives in average, although it obtained one false positive in two cases.

These results can be explained by the fact that, in many cases, the Bayesian error does not decrease by adding features, even that such features offer some gain of information about the target behavior (i.e., the average entropy decreases). Table 3 refers to an example where the prior Bayesian error of the target $Y$ is not improved by including the feature $X\left(\operatorname{CoD}_{Y}(X)=0\right)$, while the prior entropy of $Y$ is decreased by the information of $X\left(U C_{Y}(X)=0.065\right)$. Figure 1 illustrates this same example in graphics with the Bayesian error and entropy values for $P(Y=1), P(Y=1 \mid X=0)$ and $P(Y=1 \mid X=1)$. As $P(X=0)=P(X=1)$, the mean conditional entropy of $Y$ given $X$ becomes smaller than the prior entropy, since $|H(Y \mid X=1)-H(Y)|$ is smaller than $|H(Y \mid X=0)-H(Y)|$, which leads to a positive $U C_{Y}(X)$. On the other hand, since $|\varepsilon(Y \mid X=1)-\varepsilon(Y)|=|\varepsilon(Y \mid X=0)-\varepsilon(Y)|$, the Bayesian error of $Y$ given $X$ remains the same as the prior Bayesian error, which leads to $\operatorname{CoD}_{Y}(X)=0$. 
Table 3. Example that illustrates a case where $C o D_{Y}(X)=0$ and $U C_{Y}(X)>0$. (a) Probability distribution of the target $Y(\varepsilon(Y)=0.2$ and $H(Y)=0.722$ ); (b) Joint probability distribution of $X$ and $Y\left(\varepsilon(Y \mid X)=0.2 \rightarrow C o D(Y \mid X)=\frac{0.2-0.2}{0.2}=0\right.$ and $\left.H(Y \mid X)=0.675 \rightarrow U C_{Y}(X)=\frac{0.722-0.675}{0.722}=0.065\right)$.

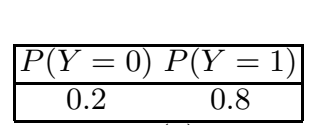

(a)

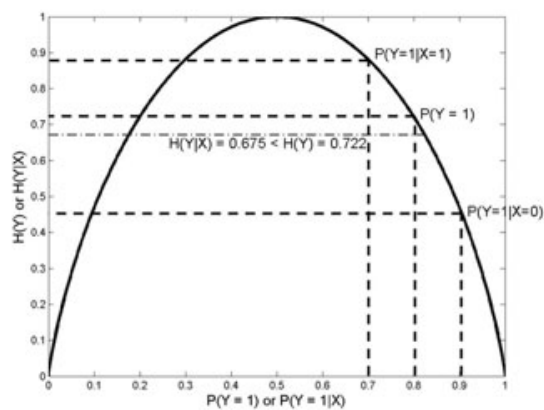

(a) Entropy

\begin{tabular}{|c|cc|}
\hline$X$ & $P(X, Y=0)$ & $P(X, Y=1)$ \\
\hline 0 & 0.05 & 0.45 \\
1 & 0.15 & 0.35 \\
\hline
\end{tabular}

(b)

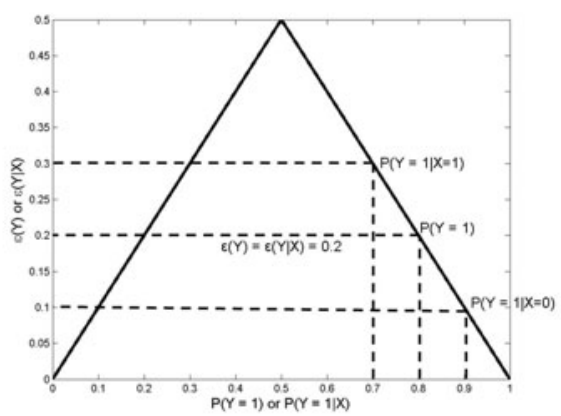

(b) Bayesian error

Fig. 1. These graphics illustrate why the mean conditional entropy of $Y$ given $X$ is smaller than the prior entropy of $Y$ and the Bayesian error of $Y$ given $X$ is the same as the prior Bayesian error of $Y$ for the joint probability distributions presented on Table 3 .

\section{Conclusion}

Considering an ideal setting where all state transition probabilities of a gene regulatory network are known, experiments using stochastic restricted Boolean networks have shown that the adoption of a feature selection criterion based on conditional entropy (uncertainty coefficient) performed a significantly better inference than that obtained by the application of a Bayesian error based criterion (non-linear coefficient of determination). This happens because, in many situations, the prior error of the target feature is not improved by the knowledge of other features even that there is some information provided by such features (i.e. the prior entropy decreases). More importantly, the SFS algorithm had very nice results when guided by the uncertainty coefficient, which could be an indicative that this approach may be used to accurately infer a network containing thousands of genes in a small time interval, even when hubs are present, i.e. genes influenced by five or more genes.

However, real microarray experiments do not provide all state transition probabilities. In fact, only a few dozens of samples are available and the error estimation becomes an important issue to address. Certainly, inference of networks 
from limited number of data samples could imply a qualitative change in the results presented here. In this sense, a future step of this research is to consider the same comparison in small sets of temporal gene expression signals. But, in principle, there is no reason to think that entropy-based criteria could perform worse than Bayesian error criteria in such situations.

We also plan to extend the comparison to more general stochastic Boolean networks, since the restricted Boolean networks do not allow some specific Boolean logic functions that can not be expressed by linear functions (e.g. exclusive-or and its negated version, considering logics with two predictor features).

\section{Acknowledgments}

This work was supported by FAPESP, CNPq and CAPES.

\section{References}

1. Sima, C., Hua, J., Jung, S.: Inference of gene regulatory networks using time-series data: A survey. Current Genomics 10, 416-429 (2009)

2. Zhang, Y., Qian, M., Ouyang, Q., Deng, M., Li, F., Tang, C.: Stochastic model of yeast cell-cycle network. Physica D 219(1), 35-39 (2006)

3. Somol, P., Pudil, P.: Fast branch \& bound algorithms for optimal feature selection. Pattern Analysis and Machine Intelligence 26(7), 900-912 (2004)

4. Ris, M., Martins Jr., D.C., Barrera, J.: U-curve: A branch-and-bound optimization algorithm for $\mathrm{u}$-shaped cost functions on boolean lattices applied to the feature selection problem. Pattern Recognition 43(3), 557-568 (2010)

5. Somol, P., Pudil, P., Novovicová, J., Paclk, P.: Adaptive floating search methods in feature selection. Pattern Recognition Letters 20, 1157-1163 (1999)

6. Martins Jr., D.C., Braga-Neto, U., Hashimoto, R.F., Dougherty, E.R., Bittner, M.L.: Intrinsically multivariate predictive genes. IEEE Journal of Selected Topics in Signal Processing 2(3), 424-439 (2008)

7. Anastassiou, D.: Computational analysis of the synergy among multiple interacting genes. Molecular Systems Biology 3(83) (2007)

8. Pudil, P., Novovičová, J., Kittler, J.: Floating search methods in feature-selection. Pattern Recognition Letters 15(11), 1119-1125 (1994)

9. Hashimoto, R.F., Kim, S., Shmulevich, I., Zhang, W., Bittner, M.L., Dougherty, E.R.: Growing genetic regulatory networks from seed genes. Bioinformatics 20(8), 1241-1247 (2004)

10. Dougherty, E.R., Brun, M., Trent, J., Bittner, M.L.: A conditioning-based model of contextual regulation. IEEE/ACM Transactions on Computational Biology and Bioinformatics (August 2007)

11. Ghaffari, N., Ivanov, I., Qian, X., Dougherty, E.R.: A CoD-based reduction algorithm for designing stationary control policies on boolean networks. Bioinformatics 26(12), 1556-1563 (2010)

12. Liang, S., Fuhrman, S., Somogyi, R.: Reveal: a general reverse engineering algorithm for inference of genetic network architectures. In: Pacific Symposium Biocomputing, PSB, pp. 18-29 (1998) 
13. Zhao, W., Serpedin, E., Dougherty, E.R.: Inferring connectivity of genetic regulatory networks using information-theoretic criteria. IEEE/ACM Transactions on Computational Biology and Bioinformatics 5(2), 262-274 (2008)

14. Theil, H.: Statistical Decomposition Analysis. North-Holland Publishing Company, Amsterdam (1972)

15. Li, F., Long, T., Lu, Y., Ouyang, Q., Tang, C.: The yeast cell-cycle network is robustly designed. Proc. Natl. Acad. Sci. USA 101(14), 4781-4786 (2004)

16. Higa, C., Hashimoto, R., Hirata, R., Hirata, N., Santos, C.: Inference of gene regulatory network using temporal coefficient of determination obtained from ergodic markov chains. In: IEEE International Workshop on Genomic Signal Processing and Statistics, GENSIPS 2009, pp. 1-4 (May 2009) 